\title{
Gender Differences in the Long-Chain Polyunsaturated Fatty Acid Status: Systematic Review of 51 Publications
}

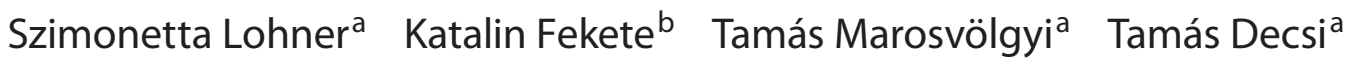 \\ Departments of a Pediatrics and ${ }^{b}$ Biochemistry and Medical Chemistry, University of Pécs, Pécs, Hungary
}

\author{
Key Words \\ Arachidonic acid · Docosahexaenoic acid $\cdot$ Gender \\ differences • Long-chain polyunsaturated fatty acids • \\ Sex hormones
}

\begin{abstract}
Background/Aims: Sex hormones may influence the activity of enzymes which are involved in the synthesis of longchain polyunsaturated fatty acids. The objective of this review was to assess the role of gender in determining the fatty acid composition of human samples, like plasma and erythrocyte membrane lipids, and adipose tissue. Methods: The method included a structured search strategy on MEDLINE, Scopus and the Cochrane databases, with formal inclusion/exclusion criteria, data extraction procedure and metaanalysis. Results: We evaluated 51 publications, dated from 1975 to 2011. Meta-analysis showed significantly lower values of both arachidonic acid (AA) and docosahexaenoic acid (DHA) in total plasma lipids (32 and 33 studies) and in plasma phospholipids (PL; 21 and 23 studies) in men than in women. Primary analysis of the phospholipid fraction showed the mean difference in AA to be $0.42 \%$ weight/weight $(95 \% \mathrm{Cl}$ : $0.18-0.65, \mathrm{n}=7,769)$ and in DHA $0.37 \%$ weight/weight $(95 \%$ Cl: 0.24-0.51, $\mathrm{n}=8,541)$, while there was no gender difference in the values of linoleic acid and $\alpha$-linolenic acid. Conclusions: This systematic review based on 51 publications
\end{abstract}

showed significantly lower contribution of AA and DHA to plasma total lipids and plasma PL in men than in women. Gender distribution should be regarded as a significant potential confounding factor in every study assessing data on fatty acid composition.

Copyright $\odot 2013$ S. Karger AG, Basel

\section{Introduction}

Long-chain-polyunsaturated fatty acids (LCPUFA) have important functions in cell membranes as indispensable building stones for human development and optimal health. Docosahexaenoic acid (DHA) and arachidonic acid (AA) are considered to be the most important functional LCPUFA. They can be provided directly from the diet or can be synthetized from their essential fatty acid precursors $\alpha$-linolenic acid (ALA) and linoleic acid (LA). Among other enzymes, $\Delta^{6}$ - and $\Delta^{5}$-desaturases are required for the formation of the longer-chain metabolites of both $n-3$ and $n-6$ series. The competitive desaturation of the $n-3$ and $n-6$ series of fatty acids by $\Delta^{6}$ desaturase is of major significance, because this step is considered to be the rate-limiting step of the pathway. The activity of this enzyme is modulated by hormones and by interactions of substrates and metabolic products $[1,2]$.

\section{KARGER \\ Fax +4161306 1234 \\ E-Mail karger@karger.ch}

www.karger.com (c) 2013 S. Karger AG, Basel

0250-6807/13/0622-0098\$38.00/0

Accessible online at:

www.karger.com/anm
Dr. Tamás Decsi

Department of Pediatrics, University of Pécs

József A. u. 7

HU-7623 Pécs (Hungary)

E-Mail tamas.decsi@aok.pte.hu 
The role of diet in influencing the proportion of fatty acids in plasma, blood cells and adipose tissue has been described in detail previously [3]. However, there are only narrative overviews [4], but no systematic review on the role of the most basic non-dietary determinant, gender, in influencing the fatty acid composition of different tissues. In order to investigate whether LCPUFA metabolism can be eventually influenced by sexual hormones, we decided to systematically review the gender-specific differences between women and men in the contribution of LCPUFA to the fatty acid composition of different biological samples.

Plasma phospholipids (PL), plasma cholesteryl esters (CE), plasma triacylglycerols (TG), total plasma lipids, erythrocyte and platelet lipids as well as subcutaneous adipose tissue from the abdomen or buttock are the lipid pools most often reported in the literature for the characterization of fatty acid status. Therefore, we decided to review data on gender differences in the fatty acid composition of these biomarkers.

\section{Methods}

\section{Aim}

The aim of this systematic literature review was to compare LCPUFA status on the basis of the fatty acid composition of different biomarkers in healthy males and females.

\section{Inclusion Criteria}

To be included into the review, a study needed to meet all of the following characteristics: (1) a study carried out in humans; (2) at least 14 participants included; (3) n-3 or n-6 LCPUFA status is reported in both males and females; (4) healthy individuals with normal weight were included, or population-based surveys were carried out in that the majority of participants were considered healthy; (5) omnivorous participants were included; (6) there was no dietary intervention (especially no lipid-modified diet) or drug therapy before sample collection, and (7) investigators measured at least 12 fatty acids by gas liquid chromatography, so the percentage distribution data contained the principal fatty acids of the fatty acid spectrum and presumably reflected a realistic proportion of fatty acids in the given lipid fraction.

\section{Search Strategy}

Electronic Searches

Ovid MEDLINE (www.ovid.com), Scopus (www.scopus. elsevier.com) and the Cochrane Library central database (www. thecochranelibrary.org) were searched from inception to February 2011 for studies containing LCPUFA values of both men and women (boys and girls) using text terms with appropriate truncation and relevant indexing terms. The search was in the form [n-3 LCPUFA terms] or [n-6 LCPUFA terms] and [biomarker terms] and [gender terms] and [differ*] and [human studies]. The results obtained by the full Ovid MEDLINE search strategy are shown in online supplementary table 1 (for all online suppl. material, see www.karger.com/doi/10.1159345599). The searches of the two other databases were also based on this strategy. We did not apply any language restriction.

\section{Data Extraction}

Titles and abstracts found by the electronic and bibliographic searches were screened for inclusion by a single reviewer (S.L.). Due to the large number of papers which were present in more than one database, duplications were filtered out to compile the final list of titles and abstracts to be screened. Thereafter, it was checked which of the titles and abstracts met the predetermined eligibility criteria. If articles seemed to meet the inclusion criteria, or the title and the abstract left room for doubt, the full text of the article was evaluated by two independent reviewers (S.L. and K.F.). If the two reviewers disagreed about the eligibility, the study was discussed in detail to reach a consensus decision.

Data for each study included were extracted by a single reviewer (S.L.) into a Microsoft Office Excel 2007 database file. To provide a standardized format, units of measurement were recalculated to percentage contribution of LCPUFA to total fatty acid composition of the relevant tissue (\% weight/weight) from the original data in the publication. If it was not possible to convert data, we tried to contact the authors. If the authors could not be contacted (in most of the cases because of the long time elapsed since the publication of the papers), or the data were not available in the original form any more, those studies were excluded. The original forms in which fatty acid data in the included studies were expressed are shown in table 1 . In some studies, the investigators measured the fatty acid composition in plasma, in other studies in serum. Because we feel that there is no major difference in the percent fatty acid composition of plasma and serum, for the uniformity of discussion, we used the term 'plasma' throughout the paper.

\section{Statistical Analyses}

Statistical analyses were performed using the Review Manager 5.1 software (Cochrane Collaboration, Oxford, UK). Mean differences (MD) were used for the analysis of continuous data. The confidence interval (CI) was established at $95 \%$. Values of $\mathrm{p}<0.05$ were considered to indicate statistical significance. Statistical heterogeneity was assessed using $\mathrm{I}^{2}$ statistics ( $\mathrm{I}^{2}$ of $50 \%$ or more indicating presence of heterogeneity).

\section{Results}

\section{Study Inclusion}

The flow diagram of the literature search for this review is shown in figure 1 . Altogether 7,925 titles and abstracts were identified via the electronic search or were found in the reference lists of the review articles. Three hundred and fifty-six of them appeared to be potentially relevant, so we attempted to collect them as full text articles. Thirteen full text articles (3.6\%) could not be collected even after repeated attempts by the help of professional librarians, but 343 full text articles were available 
Table 1. Basic characteristics of the studies included

\begin{tabular}{|c|c|c|c|c|c|c|c|c|}
\hline \multirow{2}{*}{$\begin{array}{l}\text { First author } \\
\text { publication } \\
\text { year }\end{array}$} & \multicolumn{5}{|c|}{ Characteristics of the participants } & \multirow[t]{2}{*}{ Biomarkers reported } & \multirow[t]{2}{*}{ Fatty acids reported } & \multirow{2}{*}{$\begin{array}{l}\text { Original } \\
\text { expression } \\
\text { of data }\end{array}$} \\
\hline & Country & $\begin{array}{l}\text { male } \\
\text { subjects } \\
\text { included } \\
\mathrm{n}\end{array}$ & $\begin{array}{l}\text { female } \\
\text { subjects } \\
\text { included } \\
\mathrm{n}\end{array}$ & $\begin{array}{l}\text { age of } \\
\text { males } \\
\text { years }\end{array}$ & $\begin{array}{l}\text { age of } \\
\text { females } \\
\text { years }\end{array}$ & & & \\
\hline $\begin{array}{l}\text { Ando } \\
{[5], 1990}\end{array}$ & Japan & $\begin{array}{l}31 \\
29 \\
37\end{array}$ & $\begin{array}{l}68 \\
45 \\
22\end{array}$ & $\begin{array}{l}54.3 \pm 7.4 \\
59.8 \pm 4.9 \\
64.9 \pm 7.3\end{array}$ & $\begin{array}{l}57.0 \pm 8.6 \\
59.0 \pm 6.0 \\
68.3 \pm 8.8\end{array}$ & plasma total lipids & LA, DHGLA, AA, EPA, DHA & $\begin{array}{l}\mathrm{mg} / \mathrm{dl} \\
\text { mean } \pm \mathrm{SD}\end{array}$ \\
\hline $\begin{array}{l}\text { Antonini } \\
{[6], 1970}\end{array}$ & Italy & 11 & 11 & $29 \pm 6.6$ & $27.4 \pm 6.2$ & adipose tissue & LA & $\begin{array}{l}\mathrm{w} / \mathrm{w} \% \\
\text { mean } \pm \mathrm{SD}\end{array}$ \\
\hline $\begin{array}{l}\text { Araki } \\
{[7], 1990}\end{array}$ & Japan & $\begin{array}{l}27 \\
57\end{array}$ & $\begin{array}{l}110 \\
121\end{array}$ & $\begin{array}{l}20-49 \\
50-79\end{array}$ & $\begin{array}{l}20-49 \\
50-79\end{array}$ & plasma total lipids & LA, AA, EPA, DHA & $\begin{array}{l}\mathrm{w} / \mathrm{w} \% \\
\text { mean } \pm \mathrm{SD}\end{array}$ \\
\hline $\begin{array}{l}\text { Bakewell } \\
{[8], 2006} \\
\end{array}$ & UK & 13 & 23 & $26 \pm 5$ & $23 \pm 4$ & $\begin{array}{l}\text { plasma: total lipids, } \\
\text { TG, FFA, PC, CE }\end{array}$ & $\begin{array}{l}\text { LA, GLA, DHGLA, AA, ALA, } \\
\text { EPA, DPA, DHA }\end{array}$ & $\begin{array}{l}\mathrm{w} / \mathrm{w} \% \\
\text { mean } \pm \mathrm{SD}\end{array}$ \\
\hline $\begin{array}{l}\text { Bolton-Smith } \\
{[9], 1997}\end{array}$ & UK (Scotland) & 2,308 & 2,049 & $40-59$ & $40-59$ & adipose tissue & LA, GLA, DHGLA, AA & $\begin{array}{l}\mathrm{w} / \mathrm{w} \% \\
\text { mean } \pm \mathrm{SD}\end{array}$ \\
\hline $\begin{array}{l}\text { Brouwer } \\
{[10], 1997}\end{array}$ & $\begin{array}{l}\text { The Netherlands } \\
\text { (Curacao) }\end{array}$ & 51 & 26 & $56 \pm 8$ & $58 \pm 5$ & plasma CE & $\begin{array}{l}\text { LA, GLA, DHGLA, AA, ALA, } \\
\text { EPA, DHA }\end{array}$ & $\begin{array}{l}\text { mol } \% \\
\text { mean } \pm S D\end{array}$ \\
\hline \multirow{2}{*}{$\begin{array}{l}\text { Cheng } \\
{[11], 2003}\end{array}$} & ROC (Taiwan) & & & & & & & \\
\hline & $\begin{array}{l}\text { urban } \\
\text { rural }\end{array}$ & $\begin{array}{l}10 \\
10\end{array}$ & $\begin{array}{l}10 \\
10\end{array}$ & $\begin{array}{l}10-11 \\
10-11\end{array}$ & $\begin{array}{l}10-11 \\
10-11\end{array}$ & plasma TG & LA & $\begin{array}{l}\mathrm{w} / \mathrm{w} \% \\
\text { mean } \pm \mathrm{SEM}\end{array}$ \\
\hline $\begin{array}{l}\text { Christensen } \\
{[12], 1999}\end{array}$ & Denmark & 35 & 25 & $38 \pm 10$ & $38 \pm 11$ & granulocytes, platelets & $\begin{array}{l}\text { LA, AA, ALA, EPA, DPA, } \\
\text { DHA }\end{array}$ & $\begin{array}{l}\mathrm{w} / \mathrm{w} \% \\
\text { mean } \pm \mathrm{SD}\end{array}$ \\
\hline $\begin{array}{l}\text { di Giuseppe } \\
{[13], 2009}\end{array}$ & $\begin{array}{l}\text { Italy, Belgium, } \\
\text { UK }\end{array}$ & 50 & 224 & $47 \pm 1.1$ & $44 \pm 0.5$ & $\begin{array}{l}\text { plasma total lipids, } \\
\text { erythrocyte membrane }\end{array}$ & ALA, EPA, DPA, DHA & $\begin{array}{l}\text { w/w\% } \\
\text { geometric } \\
\text { mean SEM } \\
\text { or } 95 \% \mathrm{CI}\end{array}$ \\
\hline $\begin{array}{l}\text { Elizondo- } \\
\text { Montemayor } \\
{[14], 2010}\end{array}$ & Mexico & 49 & 51 & $6-12$ & $6-12$ & plasma PL & $\begin{array}{l}\text { LA, GLA, DHGLA, AA, ALA, } \\
\text { EPA, DPA, DHA }\end{array}$ & $\begin{array}{l}\mathrm{w} / \mathrm{w} \% \\
\text { mean } \pm \mathrm{SD}\end{array}$ \\
\hline $\begin{array}{l}\text { Fernandez-Real } \\
{[15], 2001}\end{array}$ & Spain & 38 & 40 & $40.1 \pm 13.3$ & $38.1 \pm 9.3$ & plasma total lipids & LA, DHGLA, AA, EPA, DHA & $\begin{array}{l}\mathrm{w} / \mathrm{w} \% \\
\text { mean } \pm \mathrm{SD}\end{array}$ \\
\hline $\begin{array}{l}\text { Geppert } \\
{[16], 2010}\end{array}$ & UK & 40 & 34 & $32.6 \pm 8.0$ & $32.7 \pm 7.3$ & $\begin{array}{l}\text { platelet PC, } \\
\text { platelet PEA }\end{array}$ & $\begin{array}{l}\text { LA, GLA, DHGLA, AA, ALA, } \\
\text { EPA, DPA, DHA }\end{array}$ & $\begin{array}{l}\mathrm{w} / \mathrm{w} \% \\
\text { mean } \pm \mathrm{SD}\end{array}$ \\
\hline $\begin{array}{l}\text { Giltay } \\
{[17], 2004}\end{array}$ & The Netherlands & 72 & 71 & $29.6 \pm 12.9$ & $27.4 \pm 10.2$ & plasma CE & $\begin{array}{l}\text { LA, GLA, DHGLA, AA, ALA, } \\
\text { EPA, DHA }\end{array}$ & $\begin{array}{l}\text { w/w\%, } \\
\text { mean, 95\% CI }\end{array}$ \\
\hline $\begin{array}{l}\text { Glew } \\
{[18], 2010}\end{array}$ & Nigeria & 22 & 29 & $55.5 \pm 13.5$ & $47.6 \pm 8.3$ & plasma PL & $\begin{array}{l}\text { LA, GLA, DHGLA, AA, ALA, } \\
\text { EPA, DPA, DHA }\end{array}$ & $\begin{array}{l}\mathrm{w} / \mathrm{w} \% \\
\text { mean } \pm \mathrm{SD}\end{array}$ \\
\hline $\begin{array}{l}\text { Glew } \\
{[19], 2002} \\
\end{array}$ & Nigeria & 37 & 36 & 14 & 13 & plasma PL & $\begin{array}{l}\text { LA, GLA, DHGLA, AA, ALA, } \\
\text { EPA, DPA, DHA }\end{array}$ & $\begin{array}{l}\mathrm{w} / \mathrm{w} \% \\
\text { mean } \pm \mathrm{SD}\end{array}$ \\
\hline $\begin{array}{l}\text { Hagenfeldt } \\
{[20], 1975}\end{array}$ & Sweden & 6 & 8 & $26-35$ & $23-31$ & plasma FFA & LA & $\begin{array}{l}\mathrm{w} / \mathrm{w} \% \\
\text { mean } \pm \mathrm{SEM}\end{array}$ \\
\hline $\begin{array}{l}\text { Hirai } \\
{[21], 2005}\end{array}$ & Japan & 76 & 76 & $68.6 \pm 10.6$ & $67.8 \pm 11.2$ & plasma total lipids & $\begin{array}{l}\text { LA, GLA, DHGLA, AA, ALA, } \\
\text { EPA, DPA, DHA }\end{array}$ & $\begin{array}{l}\mu \mathrm{g} / \mathrm{ml} \\
\text { mean } \pm \mathrm{SD}\end{array}$ \\
\hline $\begin{array}{l}\text { Hirai } \\
{[22], 2000}\end{array}$ & $\begin{array}{l}\text { Japan, } \\
\text { The Netherlands }\end{array}$ & $\begin{array}{l}33 \\
20 \\
\end{array}$ & $\begin{array}{l}29 \\
19 \\
\end{array}$ & $\begin{array}{l}\text { university } \\
\text { students }\end{array}$ & $\begin{array}{l}\text { university } \\
\text { students }\end{array}$ & plasma total lipids & LA, AA, ALA, EPA, DHA & $\begin{array}{l}\mathrm{mg} / 100 \mathrm{ml} \\
\text { mean } \pm \mathrm{SD}\end{array}$ \\
\hline $\begin{array}{l}\text { Hodge } \\
{[23], 2007}\end{array}$ & $\begin{array}{l}\text { Australia, UK, } \\
\text { Italy, Greece }\end{array}$ & 2,048 & 2,391 & $55 \pm 8.8$ & $54.4 \pm 8.5$ & plasma PL & LA, AA, ALA, EPA, DHA & $\begin{array}{l}\mathrm{w} / \mathrm{w} \% \\
\text { mean } \pm \mathrm{SD}\end{array}$ \\
\hline $\begin{array}{l}\text { Innis } \\
{[24], 1988}\end{array}$ & Vancouver group & $\begin{array}{l}11 \\
9 \\
41 \\
14 \\
12\end{array}$ & $\begin{array}{l}17 \\
12 \\
59 \\
13 \\
12\end{array}$ & $\begin{array}{r}11-15 \\
16-20 \\
21-50 \\
>50 \\
21-50\end{array}$ & $\begin{array}{r}11-82 \\
16-20 \\
21-50 \\
>50 \\
21-50\end{array}$ & $\begin{array}{l}\text { erythrocyte PC, } \\
\text { erythrocyte PEA }\end{array}$ & $\begin{array}{l}\text { LA, GLA, DHGLA, AA, ALA, } \\
\text { EPA, DPA, DHA }\end{array}$ & $\begin{array}{l}\mathrm{w} / \mathrm{w} \% \\
\text { mean } \pm \mathrm{SEM}\end{array}$ \\
\hline $\begin{array}{l}\text { Iwamoto } \\
{[25], 2002}\end{array}$ & Japan & 20 & 20 & adults & adults & plasma CE & LA, AA, EPA, DHA & $\begin{array}{l}\text { mol } \% \\
\text { mean } \pm S E M\end{array}$ \\
\hline
\end{tabular}


Table 1 (continued)

\begin{tabular}{|c|c|c|c|c|c|c|c|c|}
\hline \multirow{2}{*}{$\begin{array}{l}\text { First author } \\
\text { publication } \\
\text { year }\end{array}$} & \multicolumn{5}{|c|}{ Characteristics of the participants } & \multirow[t]{2}{*}{ Biomarkers reported } & \multirow[t]{2}{*}{ Fatty acids reported } & \multirow{2}{*}{$\begin{array}{l}\text { Original } \\
\text { expression } \\
\text { of data }\end{array}$} \\
\hline & Country & $\begin{array}{l}\text { male } \\
\text { subjects } \\
\text { included } \\
n\end{array}$ & $\begin{array}{l}\text { female } \\
\text { subjects } \\
\text { included } \\
\mathrm{n}\end{array}$ & $\begin{array}{l}\text { age of } \\
\text { males } \\
\text { years }\end{array}$ & $\begin{array}{l}\text { age of } \\
\text { females } \\
\text { years }\end{array}$ & & & \\
\hline $\begin{array}{l}\text { Jagannathan } \\
{[26], 1969}\end{array}$ & India & 27 & 15 & $22-50$ & $26-45$ & adipose tissue & LA & $\begin{array}{l}\mathrm{w} / \mathrm{w} \% \\
\text { mean } \pm \mathrm{SEM}\end{array}$ \\
\hline $\begin{array}{l}\text { Kale } \\
{[27], 2008}\end{array}$ & India & 25 & 21 & $33.6 \pm 9.7$ & $35.1 \pm 8.0$ & erythrocyte membrane & $\begin{array}{l}\text { LA, GLA, AA, ALA, EPA, } \\
\text { DPA, DHA }\end{array}$ & $\begin{array}{l}\mathrm{w} / \mathrm{w} \% \\
\text { mean } \pm \mathrm{SD}\end{array}$ \\
\hline $\begin{array}{l}\text { Karlsson } \\
{[28], 2006}\end{array}$ & Sweden & 6 & 9 & $11.3-15.4$ & $11.7-14.5$ & plasma PL & $\begin{array}{l}\text { LA, GLA, DHGLA, AA, ALA, } \\
\text { EPA, DHA }\end{array}$ & $\begin{array}{l}\text { mol } \% \\
\text { mean } \pm S D\end{array}$ \\
\hline $\begin{array}{l}\text { Kieu } \\
{[29], 2002}\end{array}$ & $\begin{array}{l}\text { South Vietnam } \\
\text { rural } \\
\text { suburban } \\
\text { urban }\end{array}$ & $\begin{array}{l}32 \\
40 \\
39\end{array}$ & $\begin{array}{l}68 \\
58 \\
59 \\
\end{array}$ & $\begin{array}{l}47.5 \pm 5.5 \\
46.6 \pm 5.0 \\
46.4 \pm 4.7\end{array}$ & $\begin{array}{l}47.5 \pm 5.2 \\
47.6 \pm 6.4 \\
47.2 \pm 5.9\end{array}$ & plasma total lipids & $\begin{array}{l}\text { LA, GLA, DHGLA, AA, ALA, } \\
\text { EPA, DPA, DHA }\end{array}$ & $\begin{array}{l}\mathrm{w} / \mathrm{w} \% \\
\text { mean } \pm \mathrm{SD}\end{array}$ \\
\hline $\begin{array}{l}\text { Kuriki } \\
{[30], 2003}\end{array}$ & Japan & 15 & 79 & $45.3 \pm 10.6$ & $47.2 \pm 8.1$ & plasma total lipids & $\begin{array}{l}\text { LA, AA, ALA, EPA, DPA, } \\
\text { DHA }\end{array}$ & $\begin{array}{l}\mathrm{w} / \mathrm{w} \% \\
\text { mean } \pm \mathrm{SD}\end{array}$ \\
\hline $\begin{array}{l}\text { Lee } \\
{[31], 2000}\end{array}$ & Hong Kong & 81 & 113 & $43.4 \pm 11.2$ & $43.8 \pm 11.6$ & plasma total lipids & $\begin{array}{l}\text { LA, GLA, AA, ALA, EPA, } \\
\text { DHA }\end{array}$ & $\begin{array}{l}\mathrm{w} / \mathrm{w} \% \\
\text { mean } \pm \mathrm{SD}\end{array}$ \\
\hline $\begin{array}{l}\text { Lemaitre } \\
{[32], 2008}\end{array}$ & Israel & $\begin{array}{l}112 \\
49 \\
44\end{array}$ & $\begin{array}{l}118 \\
42 \\
52\end{array}$ & $\begin{array}{l}<45 \\
\quad 45-60 \\
\geq 60\end{array}$ & $\begin{array}{l}<45 \\
\quad 45-60 \\
\geq 60\end{array}$ & erythrocyte membrane & LA, DHGLA, AA, EPA, DHA & $\begin{array}{l}\mathrm{w} / \mathrm{w} \% \\
\text { mean } \pm \mathrm{SD}\end{array}$ \\
\hline $\begin{array}{l}\text { Lucas } \\
{[33], 2009}\end{array}$ & Canada & 127 & 170 & $18-74$ & $18-74$ & plasma PL & $\begin{array}{l}\text { LA, AA, ALA, EPA, DPA, } \\
\text { DHA }\end{array}$ & $\begin{array}{l}\mathrm{w} / \mathrm{w} \% \\
\text { mean } \pm \mathrm{SD}\end{array}$ \\
\hline $\begin{array}{l}\text { Mamalakis } \\
{[34], 2006}\end{array}$ & Greece & 59 & 71 & $37.7 \pm 7.9$ & $36.2 \pm 6.7$ & adipose tissue & $\begin{array}{l}\text { LA, GLA, DHGLA, AA, ALA, } \\
\text { EPA, DPA, DHA }\end{array}$ & $\begin{array}{l}\mathrm{w} / \mathrm{w} \% \\
\text { mean } \pm \mathrm{SD}\end{array}$ \\
\hline $\begin{array}{l}\text { Mamalakis } \\
{[35], 1998}\end{array}$ & Greece & 85 & 59 & $23-69$ & $23-69$ & adipose tissue & $\begin{array}{l}\text { LA, DHGLA, AA, ALA, EPA, } \\
\text { DPA, DHA }\end{array}$ & $\begin{array}{l}\mathrm{w} / \mathrm{w} \% \\
\mathrm{mean} \pm \mathrm{SD}\end{array}$ \\
\hline $\begin{array}{l}\text { McNamara } \\
{[36], 2010}\end{array}$ & USA & 10 & 10 & $35.9 \pm 8.8$ & $36.1 \pm 9.3$ & erythrocyte membrane & $\begin{array}{l}\text { LA, DHGLA, AA, EPA, DPA, } \\
\text { DHA }\end{array}$ & $\begin{array}{l}\text { w/w\% } \\
\text { mean } \pm S E M\end{array}$ \\
\hline $\begin{array}{l}\text { Melchert } \\
{[37], 1987}\end{array}$ & Germany & 38 & 70 & $21-77$ & $18-85$ & $\begin{array}{l}\text { plasma total lipids, } \\
\text { HDL } \\
\text { plasma CE } \\
\text { plasma TG } \\
\text { plasma FFA }\end{array}$ & $\begin{array}{l}\text { LA, GLA, DHGLA, AA, ALA, } \\
\text { DHA } \\
\text { LA, GLA, DHGLA, AA, ALA, } \\
\text { EPA, DHA } \\
\text { LA, GLA, AA, ALA, DHA } \\
\text { LA, DHGLA, AA, ALA, DHA }\end{array}$ & $\begin{array}{l}\mathrm{w} / \mathrm{w} \% \\
\mathrm{mean} \pm \mathrm{SD}\end{array}$ \\
\hline $\begin{array}{l}\text { Metherel } \\
{[38], 2009}\end{array}$ & Canada & 9 & 7 & $22.4 \pm 1.2$ & $22.1 \pm 1.8$ & $\begin{array}{l}\text { whole blood, } \\
\text { plasma total lipids, } \\
\text { erythrocyte membrane, } \\
\text { fingertip prick blood }\end{array}$ & $\begin{array}{l}\text { LA, GLA, DHGLA, AA, ALA, } \\
\text { EPA, DPA, DHA }\end{array}$ & $\begin{array}{l}\mathrm{w} / \mathrm{w} \% \\
\text { mean } \pm \mathrm{SD}\end{array}$ \\
\hline $\begin{array}{l}\text { Mitchell } \\
\text { [39], } 1983 \\
\end{array}$ & New Zealand & 9 & 9 & $10-13$ & $10-13$ & erythrocyte membrane & $\begin{array}{l}\text { LA, DHGLA, AA, ALA, EPA, } \\
\text { DPA, DHA }\end{array}$ & $\begin{array}{l}\mathrm{w} / \mathrm{w} \% \\
\text { mean } \pm \mathrm{SD}\end{array}$ \\
\hline $\begin{array}{l}\text { Nakamura } \\
{[40], 1995}\end{array}$ & Japan & $\begin{array}{l}18 \\
13 \\
12 \\
12\end{array}$ & $\begin{array}{l}13 \\
13 \\
15 \\
14\end{array}$ & $\begin{array}{l}30-39 \\
40-49 \\
50-59 \\
60-69\end{array}$ & $\begin{array}{l}30-69 \\
40-49 \\
50-59 \\
60-69\end{array}$ & plasma total lipids & LA, AA, EPA, DHA & $\begin{array}{l}\mathrm{w} / \mathrm{w} \% \\
\text { mean } \pm \mathrm{SD}\end{array}$ \\
\hline $\begin{array}{l}\text { Nikkari } \\
{[41], 1995}\end{array}$ & Finland & 41 & 41 & $43 \pm 4.3$ & $40 \pm 4.3$ & $\begin{array}{l}\text { plasma PL } \\
\text { plasma CE } \\
\text { plasma TG }\end{array}$ & $\begin{array}{l}\text { LA, GLA, DHGLA, AA, EPA, } \\
\text { DHA } \\
\text { LA, GLA, DHGLA, AA, ALA, } \\
\text { EPA, DHA } \\
\text { LA, AA, ALA, DHA }\end{array}$ & $\begin{array}{l}\mathrm{w} / \mathrm{w} \% \\
\text { mean } \pm \mathrm{SD}\end{array}$ \\
\hline
\end{tabular}


Table 1 (continued)

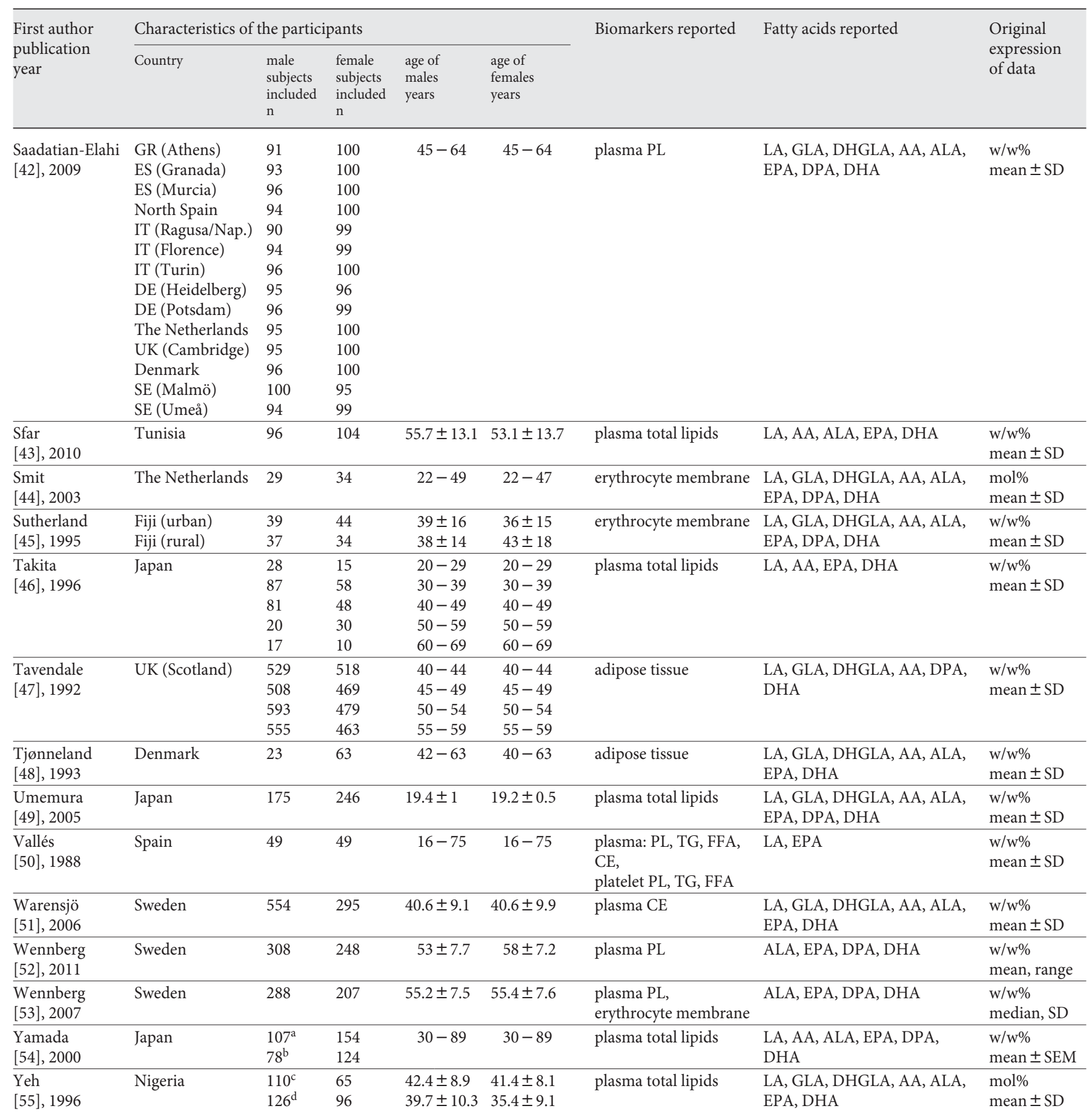
[42].

Means \pm SD and ranges. FFA $=$ Free fatty acids. Groups were classified by cluster analysis [5], age [7, 24 (only Inuits), 32, 40, 46, 47] and geographic area

${ }^{\mathrm{a}}$ Fishing group; ${ }^{\mathrm{b}}$ farming group; ${ }^{\mathrm{c}}$ senior staff group; ${ }^{\mathrm{d}}$ junior staff group. 


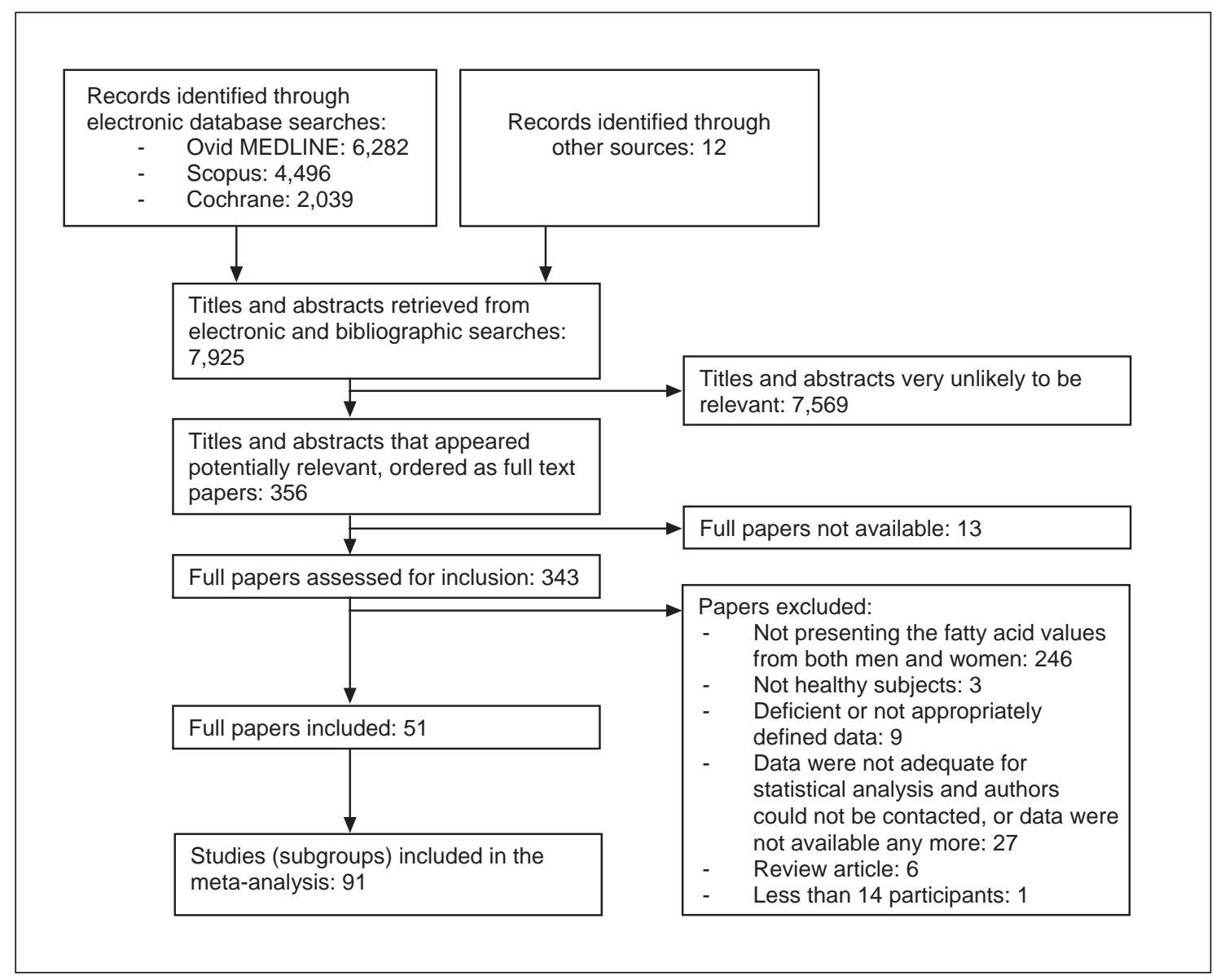

Fig. 1. Flow diagram of the systematic literature search.

for detailed assessment for inclusion. Reasons for excluding studies are presented in figure 1. In articles where the investigators analyzed the fatty acid composition of more than one biomarker (for example, plasma and erythrocyte membrane lipids), data were analyzed as originating from 2 separate studies. In some studies, the investigators divided the study population into subgroups in parallel (for example, according to age or geographical location). These subgroups were also included into our review as separate studies. Finally, 91 comparisons reported in 51 publications fulfilled the inclusion criteria.

\section{Methods Used in the Studies Reviewed}

Among the studies reviewed, there were some methodological differences in the analytical methods used for the determination of fatty acid composition of the different biomarkers. The blood samples collected were stored in a deep-frozen state, but the storage temperature was different $\left(-20,-30,-40,-70\right.$ or $\left.-80^{\circ} \mathrm{C}\right)$. Lipid extraction was carried out by chloroform and methanol in most, but not all the studies. The separation of different lipid fractions was performed by thin layer chromatography. Fatty acid analysis was carried out by gas liquid chromatography in all studies. In some studies, a packed column was used instead of capillary columns (capillary column: 34 papers; packed column: 10 papers, and column type not reported: 7 papers).

\section{Biomarkers Identified}

We found 11 publications analyzing plasma PL, whereas 8 analyzed plasma CE, 5 plasma TG, 18 total plasma lipids, 9 total erythrocyte membrane lipids, 1 platelets and 7 adipose tissue fatty acid composition. A description of biomarkers identified in 3 or more studies, including also the number of studies, subgroups and participants from both genders, and the results of the 
Table 2. Primary analysis of biomarkers identified in 3 or more studies

\begin{tabular}{|c|c|c|c|c|c|c|c|c|c|c|c|}
\hline & \multirow{2}{*}{$\begin{array}{l}\text { Indi- } \\
\text { vidual } \\
\text { stud- } \\
\text { ies, } n\end{array}$} & \multicolumn{2}{|c|}{ Participants, $\mathrm{n}$} & \multirow[t]{2}{*}{$\mathrm{MD}[95 \% \mathrm{CI}]$} & \multirow{2}{*}{$\begin{array}{l}\text { Het- } \\
\text { eroge- } \\
\text { neity } \\
\left(\mathrm{I}^{2}\right), \%\end{array}$} & & \multirow{2}{*}{$\begin{array}{l}\text { Indi- } \\
\text { vidual } \\
\text { stud- } \\
\text { ies, } n\end{array}$} & \multicolumn{2}{|c|}{ Participants, $n$} & \multirow[t]{2}{*}{$\mathrm{MD}[95 \% \mathrm{CI}]$} & \multirow{2}{*}{$\begin{array}{l}\text { Het- } \\
\text { eroge- } \\
\text { neity } \\
\left(I^{2}\right), \%\end{array}$} \\
\hline & & men & women & & & & & men & women & & \\
\hline \multicolumn{6}{|c|}{ Total plasma lipids } & \multicolumn{6}{|c|}{ Erythrocyte PEA } \\
\hline LA & 32 & 1,594 & 2,012 & $-1.55[-1.96,-1.14]^{\mathrm{a}}$ & 40 & LA & 5 & 87 & 113 & $0.26[-0.58,1.09]$ & 0 \\
\hline GLA & 11 & 738 & 881 & $-0.33[-0.07,0.00]$ & 78 & GLA & 4 & 78 & 101 & $0.02[-0.13,0.18]$ & 0 \\
\hline ALA & 18 & 1,137 & 1,614 & $-0.03[-0.06,0.00]$ & 60 & ALA & 5 & 87 & 113 & $0.08[-0.15,0.32]$ & 0 \\
\hline EPA & 32 & 1,607 & 2,166 & $0.02[-0.07,0.11]$ & 69 & EPA & 5 & 87 & 113 & $0.01[-1.10,1.13]$ & 30 \\
\hline DPA & 11 & 634 & 1,118 & $0.06[0.03,0.09]^{b}$ & 55 & $\mathrm{DPA}$ & 5 & 87 & 113 & $0.25[-0.38,0.88]$ & 6 \\
\hline DHA & 33 & 1,644 & 2,236 & $-0.12[-0.22,-0.03]^{\mathrm{a}}$ & 46 & DHA & 5 & 87 & 113 & $0.21[-1.16,1.57]$ & 0 \\
\hline \multicolumn{6}{|c|}{ Plasma PL } & \multicolumn{6}{|c|}{ Adipose tissue } \\
\hline ALA & 22 & 4,052 & 4,403 & $-0.00[-0.01,0.00]$ & 72 & ALA & 3 & 167 & 193 & $0.01[-0.02,0.03]$ & 0 \\
\hline EPA & 24 & 4,146 & 4,493 & $-0.03[-0.08,0.02]$ & 54 & EPA & 3 & 167 & 193 & $0.01[0.01,0.02]^{b}$ & 0 \\
\hline DPA & 20 & 2,002 & 2,003 & $0.01[-0.01,0.04]$ & 77 & $\mathrm{DPA}$ & 6 & 2,329 & 2,059 & $-0.01[-0.04,0.02]$ & 97 \\
\hline DHA & 23 & 4,097 & 4,444 & $-0.37[-0.51,-0.24]^{\mathrm{a}}$ & 80 & DHA & 7 & 2,352 & 2,122 & $-0.01[-0.04,0.01]$ & 96 \\
\hline
\end{tabular}

\begin{tabular}{lllllr}
\hline Plasma CE & & & & & \\
LA & 8 & 805 & 530 & $-1.03[-1.84,-0.21]^{\mathrm{a}}$ & 45 \\
GLA & 6 & 736 & 461 & $0.08[0.03,0.14]^{\mathrm{b}}$ & 32 \\
DHGLA & 6 & 736 & 461 & $0.06[0.02,0.09]^{\mathrm{b}}$ & 49 \\
AA & 7 & 756 & 481 & $0.21[-0.24,0.66]$ & 79 \\
ALA & 6 & 736 & 461 & $-0.01[-0.03,0.00]$ & 0 \\
EPA & 8 & 805 & 530 & $0.01[-0.07,0.10]$ & 55 \\
DHA & 7 & 756 & 481 & $0.02[-0.08,0.04]$ & 68 \\
\hline
\end{tabular}

\begin{tabular}{lrrrrr}
\hline Plasma TG & & & & & \\
LA & 6 & 128 & 138 & $0.35[-0.81,1.53]$ & 57 \\
AA & 3 & 59 & 69 & $0.07[-0.13,0.28]$ & 76 \\
ALA & 3 & 59 & 69 & $-0.03[-0.20,0.13]$ & 0 \\
DHA & 3 & 59 & 69 & $-0.12[-0.57,0.32]$ & 68 \\
\hline
\end{tabular}

\begin{tabular}{lrcrrr}
\hline Erythrocyte & membrane lipids & & & \\
LA & 10 & 363 & 371 & $0.05[-0.19,0.30]$ & 0 \\
GLA & 5 & 139 & 140 & $-0.00[-0.01,0.01]$ & 8 \\
DHGLA & 9 & 338 & 350 & $-0.02[-0.12,0.08]$ & 18 \\
AA & 10 & 363 & 371 & $-0.41[-0.86,0.05]$ & 64 \\
ALA & 8 & 480 & 577 & $0.01[-0.02,0.04]$ & 69 \\
EPA & 12 & 701 & 802 & $0.03[-0.00,0.07]$ & 27 \\
DPA & 9 & 495 & 590 & $0.14[-0.05,0.33]$ & 79 \\
DHA & 12 & 698 & 802 & $-0.19[-0.31,-0.06]^{\mathrm{a}}$ & 3
\end{tabular}

\begin{tabular}{lrrrrr}
\hline Erythrocyte PC & & & & \\
LA & 5 & 87 & 113 & $-0.11[-2.61,2.38]$ & 39 \\
GLA & 5 & 87 & 113 & $0.09[-0.23,0.41]$ & 43 \\
DHGLA & 5 & 87 & 113 & $-0.18[-0.40,0.04]$ & 0 \\
AA & 4 & 46 & 54 & $-1.88[-4.05,0.29]$ & 0 \\
ALA & 3 & 61 & 88 & $-0.01[-0.42,0.40]$ & 0 \\
EPA & 5 & 87 & 113 & $-0.41[-0.74,-0.08]^{\mathrm{a}}$ & 0 \\
DPA & 5 & 87 & 113 & $0.32[-0.05,0.69]$ & 0 \\
DHA & 5 & 87 & 113 & $-0.25[-1.30,0.80]$ & 62 \\
\hline
\end{tabular}

Significantly higher fatty acid values $\left({ }^{\mathrm{a}} \mathrm{p}<0.05\right)$ in women than in men and significantly higher fatty acid values $\left({ }^{b} \mathrm{p}<0.05\right)$ in men than in women are indicated.

primary analysis $\left(\mathrm{MD}, \mathrm{I}^{2}\right)$ are presented in table 2 . We discuss in detail only these biomarkers. Descriptive data of biomarkers detected in less than 3 studies are presented in table 1 .

\section{Fatty Acids Reported}

In this study, we focused on the following 8 PUFAs: LA, $\gamma$-linolenic acid (GLA), dihomo-GLA (DHGLA) and AA from the $n-6$ series and ALA, eicosapentaenoic acid (EPA), docosapentaenoic acid (DPA) and DHA from the $\mathrm{n}-3$ series. In table 1 , we list the fatty acids from these 8 if the authors included the values into the publication.

\section{Fatty Acid Composition of Total Plasma Lipids}

Primary analysis showed significantly higher contribution of the n-6 essential fatty acid, LA, and the n-6 long-chain metabolite, AA, to plasma total lipids of women compared to men (fig. 2; table 2). As to n-3 fatty acids, the values of the principal LCPUFA, DHA, were significantly higher (fig. 3), while the values of its precursor, DPA, were significantly lower in women compared to men (table 2). However, with the exception of LA and 


\begin{tabular}{|c|c|c|c|c|c|c|c|c|c|c|c|}
\hline \multirow[t]{2}{*}{ Study or subgroup } & \multicolumn{3}{|l|}{ Men } & \multicolumn{3}{|c|}{ Women } & \multirow{2}{*}{$\begin{array}{l}\text { Weight } \\
\%\end{array}$} & \multirow{2}{*}{$\begin{array}{l}\text { MD } \\
\text { IV, random }(95 \% \mathrm{Cl})\end{array}$} & \multirow{2}{*}{\multicolumn{3}{|c|}{$\begin{array}{l}\text { MD } \\
\text { IV, random, 95\% Cl }\end{array}$}} \\
\hline & mean & SD & total & mean & SD & total & & & & & \\
\hline \multicolumn{12}{|l|}{ Total plasma lipids - AA } \\
\hline Ando [5], 1990 (group I) & 6.6 & 2.06 & 31 & 6.77 & 2.12 & 68 & 2.0 & $-0.17(-1.05,0.71)$ & 1 & & \\
\hline Ando [5], 1990 (group II) & 6.76 & 2.3 & 29 & 6.88 & 2.06 & 45 & 1.6 & $-0.12(-1.15,0.91)$ & & & \\
\hline Ando [5], 1990 (group III) & 6.1 & 1.86 & 37 & 6.43 & 2.26 & 22 & 1.4 & $-0.33(-1.45,0.79)$ & & & \\
\hline Araki [7], 1990 (20-49 y) & 5.8 & 1 & 27 & 5.9 & 0.9 & 110 & 4.2 & $-0.10(-0.51,0.31)$ & & & \\
\hline Araki [7], 1990 (50-79y) & 5.2 & 1 & 57 & 5.6 & 1 & 121 & 4.8 & $-0.40(-0.71,-0.09)$ & $\rightarrow$ & & \\
\hline Bakewell [8], 2006 & 4.8 & 0.8 & 13 & 5.1 & 0.2 & 23 & 4.0 & $-0.30(-0.74,0.14)$ & $\rightarrow$ & & \\
\hline Fernandez-Real [15], 2001 & 7.3 & 1.4 & 38 & 7.2 & 1.1 & 40 & 3.3 & $0.10(-0.46,0.66)$ & - & & \\
\hline Hirai [22], 2000 (Dutch) & 6.81 & 2.1 & 20 & 7.03 & 2.14 & 19 & 1.0 & $-0.22(-1.55,1.11)$ & $\rightarrow$ & & \\
\hline Hirai [22], 2000 (Japanese) & 4.9 & 1.56 & 33 & 5.17 & 1.05 & 29 & 2.8 & $-0.27(-0.93,0.39)$ & $\rightarrow$ & - & \\
\hline Hirai [21], 2005 & 3.95 & 1 & 76 & 4.12 & 0.99 & 76 & 4.8 & $-0.17(-0.49,0.15)$ & $\rightarrow$ & & \\
\hline Kieu [29], 2002 (rural) & 6.5 & 1.4 & 39 & 6.8 & 1.5 & 59 & 3.2 & $-0.30(-0.88,0.28)$ & $\rightarrow$ & & \\
\hline Kieu [29], 2002 (suburban) & 5.9 & 1.3 & 40 & 6.2 & 1.4 & 58 & 3.4 & $-0.30(-0.84,0.24)$ & $\rightarrow$ & & \\
\hline Kieu [29], 2002 (urban) & 6.2 & 1.6 & 32 & 6.4 & 1.4 & 68 & 2.9 & $-0.20(-0.85,0.45)$ & $\rightarrow$ & & \\
\hline Kuriki [30], 2003 & 5.77 & 1.16 & 15 & 6.24 & 1.09 & 79 & 2.9 & $-0.47(-1.10,0.16)$ & $\rightarrow$ & & \\
\hline Lee [31], 2000 & 5.8 & 1.7 & 81 & 6 & 1.8 & 113 & 3.7 & $-0.20(-0.70,0.30)$ & $\rightarrow$ & & \\
\hline Melchert [37], 1987 & 6.35 & 1.28 & 37 & 6.35 & 1.36 & 70 & 3.5 & $0.00(-0.52,0.52)$ & & & \\
\hline Metherel [38], 2009 & 6.17 & 1.06 & 9 & 5.5 & 0.92 & 7 & 1.7 & $0.67(-0.30,1.64)$ & & & \\
\hline Nakamura [40], 1995 (30s) & 5.3 & 1.4 & 18 & 7.5 & 1.8 & 13 & 1.3 & $-2.20(-3.37,-1.03)$ & $\longrightarrow$ & & \\
\hline Nakamura [40], 1995 (40s) & 5.4 & 1.1 & 13 & 6.2 & 0.9 & 13 & 2.3 & $-0.80(-1.57,-0.03)$ & . & & \\
\hline Nakamura [40], 1995 (50s) & 5.7 & 1.4 & 12 & 5.8 & 2 & 15 & 1.1 & $-0.10(-1.39,1.19)$ & & & \\
\hline Nakamura [40], 1995 (60s) & 4.5 & 1.5 & 12 & 5.2 & 1.1 & 14 & 1.6 & $-0.70(-1.73,0.33)$ & & & \\
\hline Sfar [43], 2010 & 6.85 & 1.56 & 96 & 7.02 & 1.52 & 104 & 4.1 & $-0.17(-0.60,0.26)$ & & & \\
\hline Takita [46], 1996 (20-29 y) & 4.4 & 1.3 & 28 & 4.1 & 0.9 & 15 & 2.8 & $0.30(-0.36,0.96)$ & & - & \\
\hline Takita [46], 1996 (30-39 y) & 4.5 & 1.2 & 87 & 4.7 & 1 & 58 & 4.5 & $-0.20(-0.56,0.16)$ & & & \\
\hline Takita [46], 1996 (40-49 y) & 4.4 & 1.1 & 81 & 4.4 & 1.2 & 48 & 4.2 & $0.00(-0.42,0.42)$ & & & \\
\hline Takita [46], 1996 (50-59 y) & 4.4 & 1.3 & 20 & 4.9 & 1.2 & 30 & 2.6 & $-0.50(-1.21,0.21)$ & & & \\
\hline Takita [46], 1996 (60-69y) & 4.8 & 1.8 & 17 & 4.3 & 0.6 & 10 & 1.8 & $0.50(-0.43,1.43)$ & & . & \\
\hline Umemura [49], 2005 & 7.1 & 1.3 & 175 & 6.6 & 1.1 & 246 & 5.3 & $0.50(0.26,0.74)$ & & $\rightarrow$ & \\
\hline Yamada [54], 2000 (farming) & 5.1 & 1.77 & 78 & 5.5 & 1.11 & 124 & 4.0 & $-0.40(-0.84,0.04)$ & $\rightarrow$ & & \\
\hline Yamada [54], 2000 (fishing) & 5.9 & 1.03 & 107 & 6 & 1.24 & 154 & 5.1 & $-0.10(-0.38,0.18)$ & & & \\
\hline Yeh [55], 1996 (junior staff) & 4.56 & 1.22 & 126 & 5.41 & 1.42 & 96 & 4.6 & $-0.85(-1.21,-0.49)$ & $\rightarrow$ & & \\
\hline Yeh [55], 1996 (senior staff) & 5.27 & 1.4 & 110 & 5.91 & 1.94 & 65 & 3.4 & $-0.64(-1.18,-0.10)$ & & & \\
\hline \multirow{2}{*}{\multicolumn{12}{|c|}{$\begin{array}{l}\text { Subtotal }(95 \% \mathrm{Cl}) \\
\text { Heterogeneity: } \mathrm{t}^{2}=0.09 ; \mathrm{x}^{2}=77.70, \text { d.f. }=31(\mathrm{p}<0.00001) ;\left.\right|^{2}=60 \%\end{array}$}} \\
\hline & & & & & & & & & & & \\
\hline \multicolumn{7}{|c|}{ Test for overall effect: $Z=2.90(p=0.004)$} & & -4 & $-2 \quad 0$ & 2 & 4 \\
\hline \multicolumn{12}{|c|}{ Favors women } \\
\hline
\end{tabular}

Fig. 2. MD in the percent contribution of AA to total plasma lipids of healthy male and female subjects. Groups I-III [5] were classified by cluster analysis. $\mathrm{y}=$ Years.

DHA, considerable heterogeneity was seen among the results of the individual studies.

To filter out the potential effect of age, we tried to classify the individual studies into three age categories: $0-12$ (a), 13-50 (b) and $\geq 51$ years (c). In the case of plasma total lipids, classification according to age categories yielded sufficient numbers of studies to evaluate the effect of gender in the two adult groups only. Among the longchain metabolites, in the group aged $13-50$ years only the values of DHA (MD: -0.16 ; 95\% CI: $-0.26,-0.06 ; 2,418$ participants; $\mathrm{I}^{2}=45 \%$ ) were significantly higher in women, while the values of DPA were significantly lower in women compared to men (MD: 0.07; 95\% CI: 0.03, 0.01; 1,130 participants; $\left.\mathrm{I}^{2}=67 \%\right)$. In the group aged $\geq 51$ years (containing postmenopausal women), the values of the n-6 LCPUFA, DHGLA (MD: -0.14; 95\% CI: $-0.24,-0.04$; 137 participants; $\mathrm{I}^{2}=19 \%$ ) and AA (MD: -0.25 ; 95\% CI: $-0.43,-0.08 ; 892$ participants; $\mathrm{I}^{2}=0 \%$ ) were significantly higher in women compared to men, whereas DHA values did not differ between the two genders. 


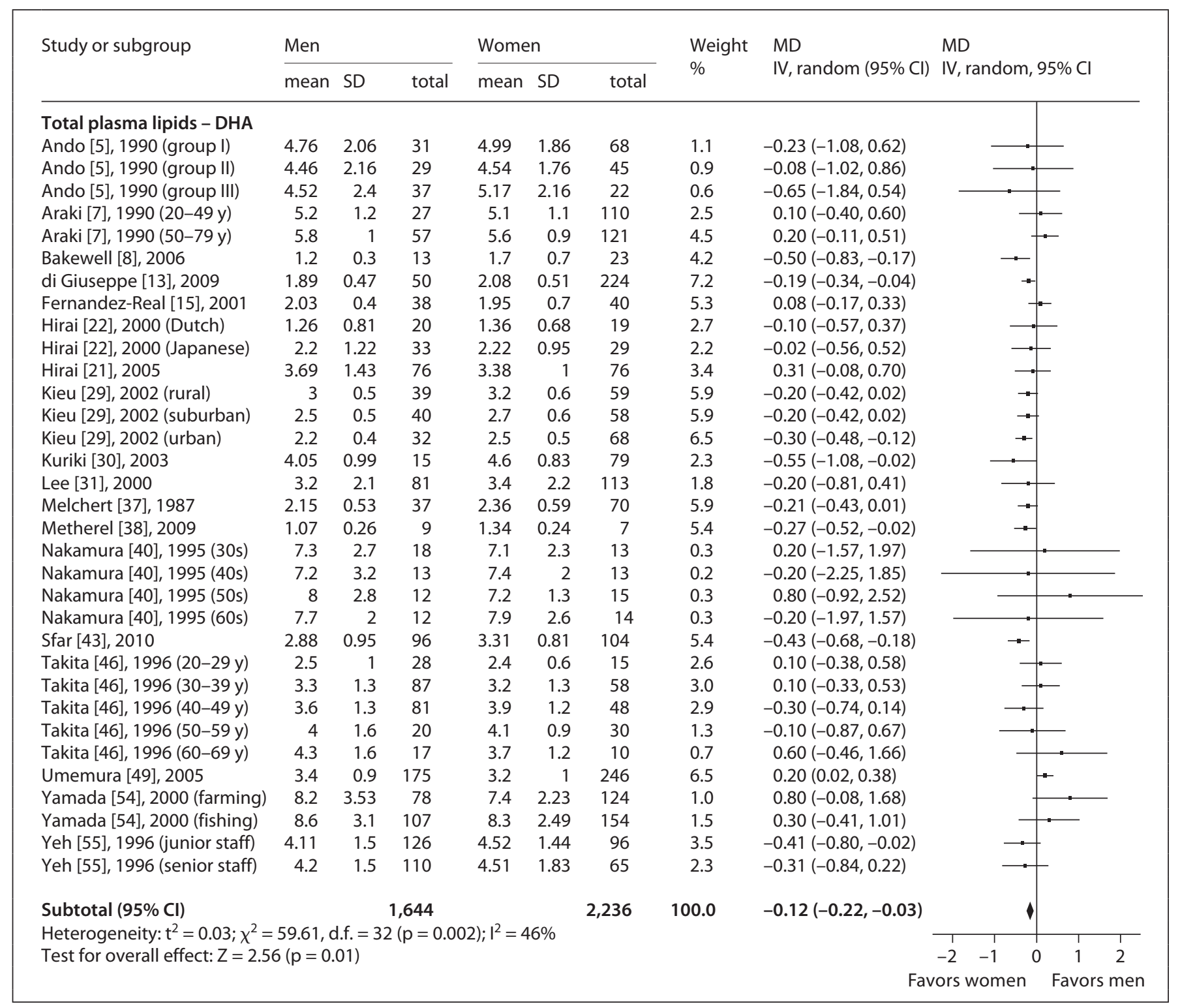

Fig. 3. MD in the percent contribution of docosahexaenoic acid to total plasma lipids of healthy male and female subjects. Groups I-3 [5] were classified by cluster analysis. y = Years.

To consider the potential effect of diet, we classified the studies based on the results of Meyer [56] according to fish eating habits, assigning the Inuit of Nunavik and the Japanese as high n-3 LCPUFA intake group and all other people as low n-3 LCPUFA intake group. In the high fish (n-3 LCPUFA) intake group, values of AA were significantly higher in women compared to men (MD: -0.23 ; 95\% CI: $-0.44,-0.02 ; 2,291$ participants; $\left.\mathrm{I}^{2}=68 \%\right)$, while there was no significant difference in the DHA levels between the two groups (MD: $-0.00 ; 95 \%$ CI: $-0.12,0.12 ; 2,291$ participants; $\left.I^{2}=17 \%\right)$. In the low fish intake group, both AA (MD: -0.24 ; 95\% CI: $-0.41,-0.07 ; 1,383$ participants; $\mathrm{I}^{2}=$ $8 \%$ ) and DHA (MD: -0.24 ; 95\% CI: $-0.31,-0.16 ; 1,598$ participants; $\mathrm{I}^{2}=11 \%$ ) were significantly higher in women.

\section{Fatty Acid Composition of Plasma PL}

Plasma PL compositional data were reported for the largest number of men $(n=4,097)$ and women $(n=4,444)$. 


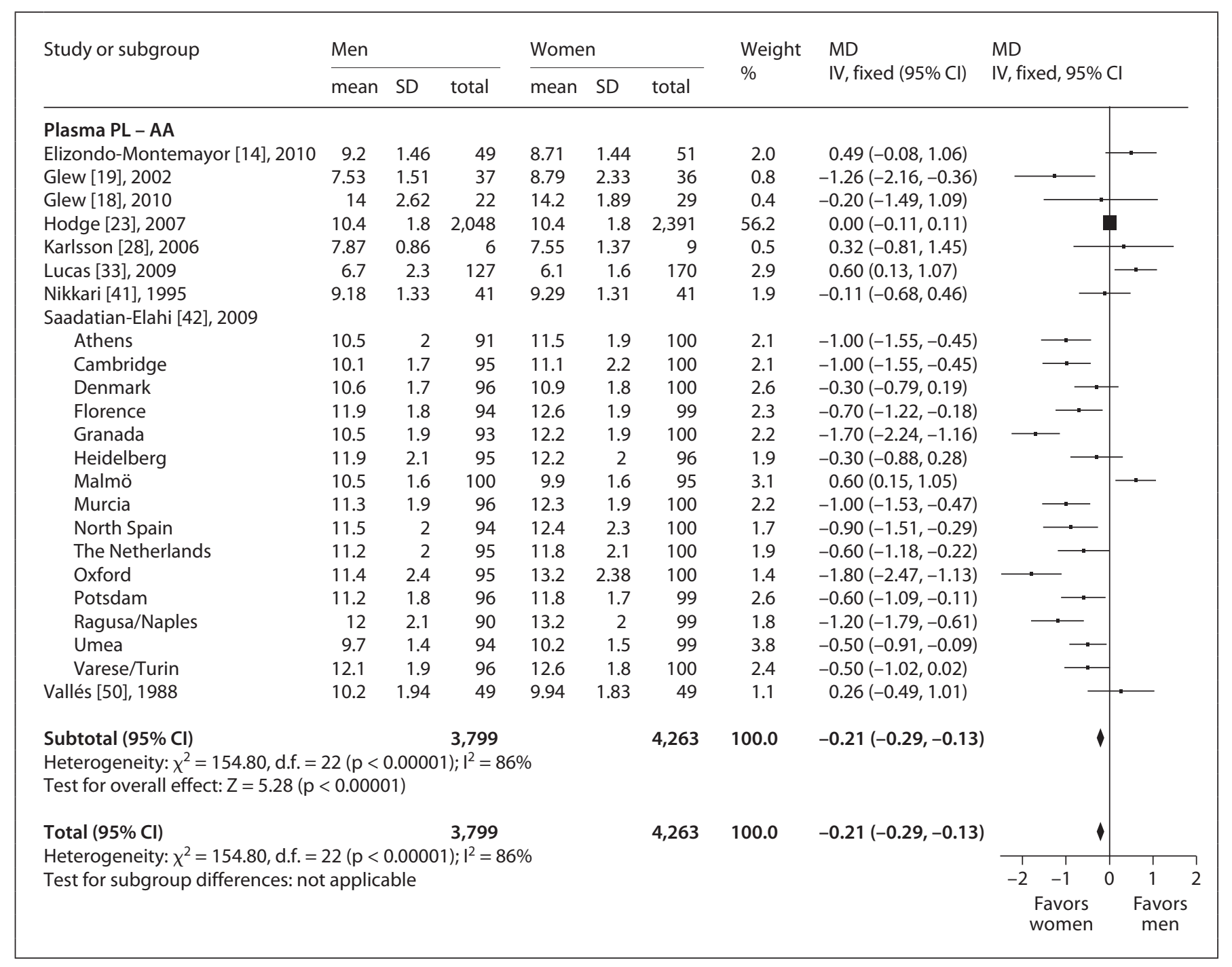

Fig. 4. $\mathrm{MD}$ in the percent contribution of AA to plasma PL of healthy male and female subjects.

There was 1 cross-sectional study among the articles [42] included that contained 16 subgroups stratified according to geographic areas; we were able to include 14 subgroups (in one area only women were recruited, whereas another subgroup had to be excluded, because there were also vegans among the participants). Primary analysis revealed significantly higher contribution of both AA (MD: -0.42; 95\% CI: $-0.65,-0.18 ; 7,769$ participants; $\mathrm{I}^{2}=81 \%$ ) and DHA (MD: -0.37 ; 95\% CI: $-0.51,-0.24 ; 8,541$ participants; $\left.\mathrm{I}^{2}=79 \%\right)$ to the fatty acid composition of plasma PL in women (fig. 4,5), while in LA and ALA there was no gender difference. There were not enough studies to carry out subgroup analysis either by age or fish eating habits.

\section{Fatty Acid Composition of Plasma CE}

Plasma CE fatty acids were reported in 8 publications. There was no gender difference in AA and DHA values (table 2), but GLA and DHGLA were found significantly higher in men as compared to women.

\section{Fatty Acid Composition of Plasma TG}

Five publications reported fatty acid composition of plasma TG in both men and women. The primary analysis showed no difference between both sexes in any of the fatty acids discussed (table 2). 


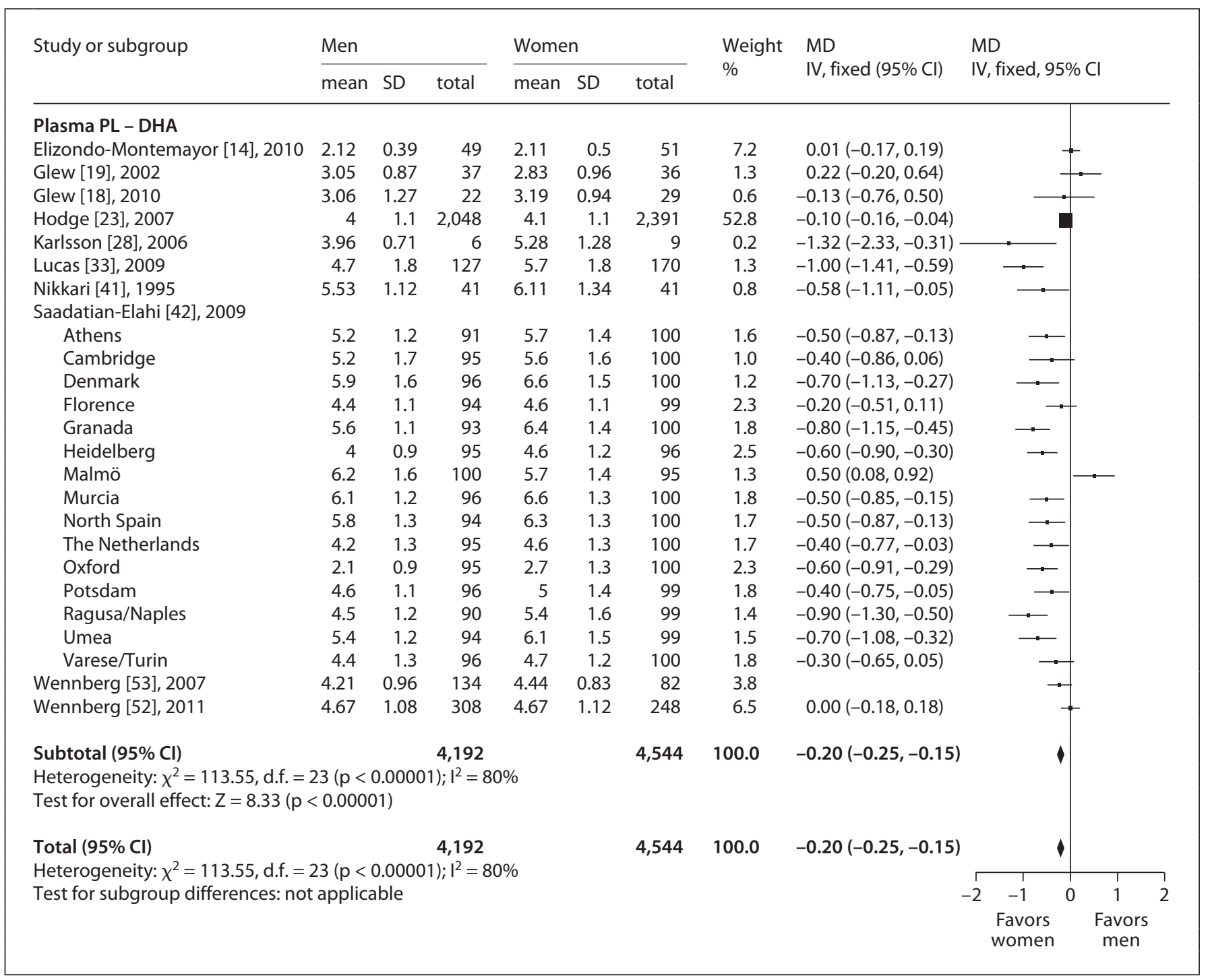

Fig. 5. MD in the percent contribution of DHA to plasma PL of healthy male and female subjects.

\section{Fatty Acid Composition of Erythrocyte Membrane Total Lipids}

There were 9 publications reporting fatty acid composition of the erythrocyte membrane total lipids in both sexes. The primary analysis showed significant differences only in DHA values, which were higher in women than in men (table 2). Because most of these studies were carried out in participants older than 50 years, in spite of the apparent abundance of data (12 studies with 1,224 participants), there was no possibility to carry out subgroup analysis either by age or fish eating habits.
Fatty Acid Composition of Erythrocyte

Phosphatidylcholine and Phosphatidylethanolamine

There was 1 paper reporting erythrocyte phosphatidylcholine (PC) and phosphatidylethanolamine (PEA) fatty acid composition in 5 subgroups [24]. In erythrocyte PC the values of EPA were significantly higher in women than in men, whereas in erythrocyte PEA there was no difference between the two sexes in any of the fatty acids discussed (table 2). 
Fatty Acid Composition of Adipose Tissue

In adipose tissue the values of LA and DHGLA were significantly higher in women than in men, while the values of GLA and AA, and from the n-3 series the values from EPA, were significantly higher in men than in women.

\section{Discussion}

Endogenous synthesis of AA and DHA from their essential fatty acid precursors, LA and ALA, requires the contribution of elongases and desaturases. Since the elongation steps are rapid, whereas the desaturation steps are slower, the latter are considered as the rate-limiting steps. In this systematic review, we found higher contributions of LCPUFA (AA and DHA) to both plasma total lipids and plasma PL in women than in men. We also found significantly higher values of DHA, but not of AA, in women than in men in erythrocyte lipids. It may be assumed with good reason that the higher AA and DHA values found in plasma PL of women may be due to the higher activity of desaturases, especially that of $\Delta^{6}$-desaturase.

The effects of sex hormones on essential fatty acid metabolism in humans have already been reviewed in detail by Childs et al. [57]. In short, human studies demonstrated that males and females differ in their ability to synthesize n-3 LCPUFA from ALA, leading to the higher circulating concentrations of DHA in women than in men [57]. In the same review, a significant relationship between plasma and tissue fatty acid composition and circulating sex hormone concentrations was seen, suggesting that estrogen stimulates, whereas testosterone inhibits, the conversion of essential fatty acids into their longer-chain metabolites. This is consistent with findings from both animal studies [58] and human stable-isotope studies, which also showed that women have a higher capacity than men to synthesize DHA from ALA $[1,59]$.

We also tried to evaluate the influence of age on the gender differences in fatty acid composition of biological samples. Since previously several authors suggested that gender differences seen in fatty acid compositional data may be due to the higher estrogen levels in women, we decided to make subgroups according to the presumably changing estrogen levels of women in the course of their life cycle: 0-12 (a), 13-50 (b) and 50-75 years (c). In serum total lipids, the higher levels of LCPUFA in women than in men were also seen in the oldest age group, where mostly postmenopausal women

Gender Differences in the LCPUFA

Status were present. This finding on its own may indicate that not only the higher estrogen levels are responsible for the sex differences in LCPUFA values observed, e.g. in addition to the physiological and hormonal changes caused by aging, different age groups can have very different dietary habits, which may also influence the composition of serum lipids. Moreover, many postmenopausal women receive estrogen supplementation therapy, which in fact may further modify the picture. The data obtained in the present study indicate that gender differences in fatty acid status may be relevant also in the elderly.

Diet is considered as the major factor influencing fatty acid composition in tissues. Plasma PL fatty acid composition represents the dietary intake of fatty acids over periods of weeks or months before sample collection [60, 61], while the rate of changes in red blood cells is slower than that seen in plasma lipids $[62,63]$. The adipose tissue fatty acid pattern represents the diet ingested in the previous $1-2.5$ years $[64,65]$. In the vast majority of the studies included into our review, the composition of diets was not investigated, so it is not possible to tell to what extent dietary fatty acids influence our results. However, there were enough studies to carry out a subgroup analysis in the total plasma lipid fraction by $n-3$ LCPUFA intake characteristic for the investigated populations. This analysis resulted in an appreciable decrease in the degree of heterogeneity, which confirms the important role of diet in determining LCPUFA status. In the high DHA intake group (including Japanese and Inuit subjects), no significant difference in DHA values was seen between men and women. This observation suggests that gender is a significant potential confounding variable mainly in populations with low dietary $n-3$ intake.

We think that our systematic review has some strongpoints. Firstly, we were able to identify a considerable number of studies investigating a relatively great number of women and men. Secondly, the studies included in the present review originated from a wide diversity of geographic locations; consequently, the results obtained may be applied without serious geographical restriction. Thirdly, the studies analyzed were carried out during more than 3 decades, so the phenomena observed do not seem to be changing over time. However, there are also some weaknesses of our study. Firstly, studies included were mostly observational studies and not randomized controlled trials, so the quality control potential of the studies included into the review was limited. Secondly, in case of plasma PL, 14 data sets (subgroups) originated 
from the same cross-sectional study, so these data influenced the statistical results notably. However, these subgroups represented 14 different geographic areas, and so there are good arguments for handling them as different studies. Thirdly, the analytical methods used by the different research groups were not standardized. However, methods of fatty acid analysis are not yet as rigorously standardized as many other laboratory methods, so any systematic review of fatty acid data faces the same difficulty. It is also important to add that there are several factors which influence fatty acid metabolism and differ between genders (e.g. dietary fatty acid intake, alcohol ingestion, relative body fatness or level of physical activity). These factors may all contribute to the gender differences observed in fatty acid composition of different biological samples; however, the main objective of the present review was not to investigate the potential contribution of these factors, but to draw attention to the fact that genderrelated differences exist.
In supplementation studies reporting fatty acid composition in serum PL, serum total lipids or erythrocyte membrane lipids, gender distribution should be regarded as significant potential confounding variable.

\section{Acknowledgments}

This study was financially supported by the Hungarian $\mathrm{Na}$ tional Research Fund (OTKA K77899) and the European Communities 7th Framework Program (NUTRIMENTHE FP7 212652). This paper does not necessarily reflect the views of the Commission of the European Communities and in no way anticipates the future policy in this area.

\section{Disclosure Statement}

The authors confirm that there are no financial or other relationships which might lead to a conflict of interest in the publication of this work.

\section{References}

$>_{1}$ Burdge GC, Wootton SA: Conversion of alpha-linolenic acid to eicosapentaenoic, docosapentaenoic and docosahexaenoic acids in young women. Br J Nutr 2002;88:411-420.

2 Harnack K, Andersen G, Somoza V: Quantitation of alpha-linolenic acid elongation to eicosapentaenoic and docosahexaenoic acid as affected by the ratio of $\mathrm{n} 6 / \mathrm{n} 3$ fatty acids. Nutr Metab (Lond) 2009;6:8.

3 Hodson L, Skeaff CM, Fielding BA: Fatty acid composition of adipose tissue and blood in humans and its use as a biomarker of dietary intake. Prog Lipid Res 2008;47:348380.

4 Decsi T, Kennedy K: Sex-specific differences in essential fatty acid metabolism. Am J Clin Nutr 2011;94:1914S-1919S.

- 5 Ando T, Yanagihashi T, Wakisaka I, Tomari T: Relationship between serum fatty acid composition and life style and/or health status in a rural population in Kagoshima (in Japanese). Nihon Koshu Eisei Zasshi 1990; 37:752-760.

6 Antonini FM, Bucalossi A, Petruzzi E, Simoni R, Morini PL, D’Alessandro A: Fatty acid composition of adipose tissue in normal, atherosclerotic and diabetic subjects. Atherosclerosis 1970;11:279-289.

7 Araki K, Akiyam Y, Murayama H, Fukase O, Yamamoto A, Fujiwara T, Oshiba K, Kawaraya $\mathrm{C}$, Otachi J, Masumoto K: Relationship between dietary intake and serum fatty acids compositions of the residents in Hyogo Prefecture (in Japanese). Nihon Koshu Eisei Zasshi 1990;37:620-628.
-8 Bakewell L, Burdge GC, Calder PC: Polyunsaturated fatty acid concentrations in young men and women consuming their habitual diets. Br J Nutr 2006;96:93-99.

-9 Bolton-Smith C, Woodward M, Tavendale R: Evidence for age-related differences in the fatty acid composition of human adipose tissue, independent of diet. Eur J Clin Nutr 1997;51:619-624.

10 Brouwer DA, van der Dijs FP, Leerink CB, Steward HN, Kroon TA, Suverkropp GH, Romer JW, van Doormaal JJ, Muskiet FA: The dietary fatty acids of patients with coronary artery disease and controls in Curacao. Implications for primary and secondary prevention. West Indian Med J 1997;46:53-56.

11 Cheng HH, Wen YY, Chen C: Serum fatty acid composition in primary school children is associated with serum cholesterol levels and dietary fat intake. Eur J Clin Nutr 2003; 57:1613-1620.

-12 Christensen JH, Christensen MS, Dyerberg J, Schmidt EB: Heart rate variability and fatty acid content of blood cell membranes: a dose-response study with n-3 fatty acids. Am J Clin Nutr 1999;70:331-337.

13 di Giuseppe R, de Lorgeril M, Salen P, Laporte F, Di Castelnuovo A, Krogh V, Siani A, Arnout J, Cappuccio FP, van Dongen M, et al: Alcohol consumption and n-3 polyunsaturated fatty acids in healthy men and women from 3 European populations. Am J Clin Nutr 2009;89:354-362.
14 Elizondo-Montemayor L, Serrano-González M, Ugalde-Casas PA, Cuello-García C, Borbolla-Escoboza JR: Plasma phospholipid fatty acids in obese male and female Mexican children. Ann Nutr Metab 2010;57:234-241.

15 Fernandez-Real JM, Vayreda M, Casamitjana R, Gonzalez-Huix F, Ricart W: Circulating granulocyte-macrophage colony-stimulating factor and serum fatty acid composition in men and women. Metabolism 2001; 50:1479-1483.

16 Geppert J, Min Y, Neville M, Lowy C, Ghebremeskel K: Gender-specific fatty acid profiles in platelet phosphatidyl-choline and -ethanolamine. Prostaglandins Leukot Essent Fatty Acids 2010;82:51-56.

17 Giltay EJ, Gooren LJ, Toorians AW, Katan MB, Zock PL: Docosahexaenoic acid concentrations are higher in women than in men because of estrogenic effects. Am J Clin Nutr 2004;80:1167-1174.

18 Glew RH, Chuang LT, Berry T, Okolie H, Crossey MJ, VanderJagt DJ: Lipid profiles and trans fatty acids in serum phospholipids of semi-nomadic Fulani in northern Nigeria. J Health Popul Nutr 2010;28:159-166.

19 Glew RH, Casados JK, Huang YS, Chuang LT, VanderJagt DJ: The fatty acid composition of the serum phospholipids of children with sickle cell disease in Nigeria. Prostaglandins Leukot Essent Fatty Acids 2002;67: 217-222.

20 Hagenfeldt L, Hagenfeldt K, Wennmalm A: Turnover of plasma free arachidonic and oleic acids in men and women. Horm Metab Res 1975;7:467-471. 
-21 Hirai K, Kozuki M, Miyanaga K, Miyagawa F, Takezoe R, Hasegawa M, Mori M: Lower levels of eicosapentaenoic acid and the ratio of docosahexaenoic acid to arachidonic acid in sera of patients with multi-infarct dementia. J Clin Biochem Nutr 2005;36:83-89.

-22 Hirai K, Horiuchi R, Ohno Y, Higuchi H, Asano Y: Lower eicosapentaenoic acid and higher arachidonic acid levels in sera of young adults in the Netherlands than in Japan. Environ Health Prev Med 2000;5:6065.

23 Hodge AM, Simpson JA, Gibson RA, Sinclair AJ, Makrides M, O’Dea K, English DR, Giles GG: Plasma phospholipid fatty acid composition as a biomarker of habitual dietary fat intake in an ethnically diverse cohort. Nutr Metab Cardiovasc Dis 2007;17:415-426.

24 Innis SM, Kuhnlein HV, Kinloch D: The composition of red cell membrane phospholipids in Canadian Inuit consuming a diet high in marine mammals. Lipids 1988;23: 1064-1068.

-25 Iwamoto M, Imaizumi K, Sato M, Hirooka Y, Sakai K, Takeshita A, Kono M: Serum lipid profiles in Japanese women and men during consumption of walnuts. Eur J Clin Nutr 2002;56:629-637.

26 Jagannathan SN: Fatty acid composition of adipose tissue in Indian adults: sex difference and influence of pregnancy. Ind J Biochem 1969;6:222-225.

-27 Kale A, Joshi S, Naphade N, Sapkale S, Raju MS, Pillai A, Nasrallah H, Mahadik SP: Opposite changes in predominantly docosahexaenoic acid (DHA) in cerebrospinal fluid and red blood cells from never-medicated first-episode psychotic patients. Schizophr Res 2008;98:295-301.

-28 Karlsson M, Marild S, Brandberg J, Lonn L, Friberg P, Strandvik B: Serum phospholipid fatty acids, adipose tissue, and metabolic markers in obese adolescents. Obesity 2006; 14:1931-1939.

-29 Kieu NT, Yasugi E, Hung NT, Kido T, Kondo K, Yamamoto S, Chuyen NV, Oshima M: Serum fatty acids, lipoprotein (a) and apolipoprotein profiles of middle-aged men and women in South Vietnam. Asia Pac J Clin Nutr 2002;11:112-116.

- 30 Kuriki K, Nagaya T, Tokudome Y, Imaeda N, Fujiwara N, Sato J, Goto C, Ikeda M, Maki S, Tajima K, et al: Plasma concentrations of (n3) highly unsaturated fatty acids are good biomarkers of relative dietary fatty acid intakes: a cross-sectional study. J Nutr 2003; 133:3643-3650.

- 31 Lee HY, Woo J, Chen ZY, Leung SF, Peng XH: Serum fatty acid, lipid profile and dietary intake of Hong Kong Chinese omnivores and vegetarians. Eur J Clin Nutr 2000;54:768773.

- 32 Lemaitre RN, Siscovick DS, Berry EM, Kark JD, Friedlander Y: Familial aggregation of red blood cell membrane fatty acid composition: the Kibbutzim Family Study. Metabolism 2008;57:662-668.
33 Lucas M, Dewailly E, Blanchet C, Gingras S, Holub BJ: Plasma omega-3 and psychological distress among Nunavik Inuit (Canada). Psychiatry Res 2009;167:266-278.

34 Mamalakis G, Kalogeropoulos N, Andrikopoulos N, Hatzis C, Kromhout D, Moschandreas J, Kafatos A: Depression and long chain n-3 fatty acids in adipose tissue in adults from Crete. Eur J Clin Nutr 2006;60: 882-888.

35 Mamalakis G, Kafatos A, Tornaritis M, Alevizos B: Anxiety and adipose essential fatty acid precursors for prostaglandin E1 and E2. J Am Coll Nutr 1998;17:239-243.

36 McNamara RK, Jandacek R, Rider T, Tso P, Dwivedi Y, Pandey GN: Selective deficits in erythrocyte docosahexaenoic acid composition in adult patients with bipolar disorder and major depressive disorder. J Affect Disord 2010;126:303-311.

37 Melchert HU, Limsathayourat N, Mihajlovic H, Eichberg J, Thefeld W, Rottka H: Fatty acid patterns in triglycerides, diglycerides, free fatty acids, cholesteryl esters and phosphatidylcholine in serum from vegetarians and non-vegetarians. Atherosclerosis 1987; 65:159-166.

38 Metherel AH, Armstrong JM, Patterson AC, Stark KD: Assessment of blood measures of n-3 polyunsaturated fatty acids with acute fish oil supplementation and washout in men and women. Prostaglandins Leukot Essent Fatty Acids 2009;81:23-29.

39 Mitchell EA, Lewis S, Cutler DR: Essential fatty acids and maladjusted behaviour in children. Prostaglandins Leukot Med 1983; 12:281-287.

40 Nakamura T, Takebe K, Tando Y, Arai Y, Yamada N, Ishii M, Kikuchi H, Machida K, Imamura K, Terada A: Serum fatty acid composition in normal Japanese and its relationship with dietary fish and vegetable oil contents and blood lipid levels. Ann Nutr Metab 1995;39:261-270.

41 Nikkari T, Luukkainen P, Pietinen P, Puska $P$ : Fatty acid composition of serum lipid fractions in relation to gender and quality of dietary fat. Ann Med 1995;27:491-498.

42 Saadatian-Elahi M, Slimani N, Chajes V, Jenab M, Goudable J, Biessy C, Ferrari P, Byrnes G, Autier P, Peeters PH, et al: Plasma phospholipid fatty acid profiles and their association with food intakes: results from a cross-sectional study within the European Prospective Investigation into Cancer and Nutrition. Am J Clin Nutr 2009;89:331-346.

-43 Sfar S, Laporte F, Braham H, Jawed A, Amor $S$, Kerkeni A: Influence of dietary habits, age and gender on plasma fatty acids levels in a population of healthy Tunisian subjects. Exp Gerontol 2010;45:719-725.

44 Smit EN, Fokkema MR, Boersma ER, Muskiet FA: Higher erythrocyte 22:6n-3 and $22: 5 n-6$, and lower 22:5n-3 suggest higher delta-4-desaturation capacity in women of childbearing age. Br J Nutr 2003;89:739-740.
45 Sutherland WH, Shilton ME, Nye ER, Gillies ME, Bakani I, Robertson MC: Urban/rural differences in red blood cell fatty acid composition, plasma lipids and diet in Melanesian Fijians. Eur J Clin Nutr 1995;49:233241.

46 Takita T, Nakamura K, Kimira M, Yamada N, Kobayashi Y, Innami S: Serum fatty acid compositions and lipid concentrations and their correlations. J Clin Biochem Nutr 1996; 20:149-159.

47 Tavendale R, Lee AJ, Smith WC, TunstallPedoe H: Adipose tissue fatty acids in Scottish men and women: results from the Scottish Heart Health Study. Atherosclerosis 1992;94:161-169.

-48 Tjønneland A, Overvad K, Thorling E, Ewertz M: Adipose tissue fatty acids as biomarkers of dietary exposure in Danish men and women. Am J Clin Nutr 1993;57:629-633.

-49 Umemura U, Ishimori M, Kobayashi T, Tamura Y, Koike KA, Shimamoto T: Possible effects of diets on serum lipids, fatty acids and blood pressure levels in male and female Japanese university students. Environ Health Prev Med 2005;10:42-47.

50 Vallés J, Aznar J, Santos MT: Composition of platelet fatty acids and their modulation by plasma fatty acids in humans: effect of age and sex. Atherosclerosis 1988;71:215-225.

51 Warensjö E, Ohrvall M, Vessby B: Fatty acid composition and estimated desaturase activities are associated with obesity and lifestyle variables in men and women. Nutr Metab Cardiovasc Dis 2006;16:128-136.

52 Wennberg M, Bergdahl IA, Hallmans G, Norberg M, Lundh T, Skerfving S, Strömberg U, Vessby B, Jansson JH: Fish consumption and myocardial infarction: a second prospective biomarker study from northern Sweden. Am J Clin Nutr 2011;93:27-36.

53 Wennberg M, Bergdahl IA, Stegmayr B, Hallmans G, Lundh T, Skerfving S, Strömberg U, Vessby B, Jansson JH: Fish intake, mercury, long-chain $n-3$ polyunsaturated fatty acids and risk of stroke in northern Sweden. Br J Nutr 2007;98:1038-1045.

54 Yamada T, Strong JP, Ishii T, Ueno T, Koyama M, Wagayama H, Shimizu A, Sakai T, Malcom GT, Guzman MA: Atherosclerosis and omega- 3 fatty acids in the populations of a fishing village and a farming village in Japan. Atherosclerosis 2000;153:469-481.

55 Yeh LL, Kuller LH, Bunker CH, Ukoli FA, Huston SL, Terrell DF: The role of socioeconomic status and serum fatty acids in the relationship between intake of animal foods and cardiovascular risk factors. Ann Epidemiol 1996;6:290-298.

56 Meyer BJ: Are we consuming enough long chain omega-3 polyunsaturated fatty acids for optimal health? Prostaglandins Leukot Essent Fatty Acids 2011;85:275-280.

57 Childs CE, Roume-Nadal, Burdge GC, Calder PC: Gender differences in the n-3 fatty acid content of tissues. Proc Nutr Soc 2008;67:19-27. 
58 Extier A, Langelier B, Perruchot MH, Guesnet P, Van Veldhoven PP, Lavialle M, Alessandri JM: Gender affects liver desaturase expression in a rat model of n-3 fatty acid repletion. J Nutr Biochem 2010;21:180187.

59 Pawlosky R, Hibbeln J, Lin Y, Salem N Jr: n-3 fatty acid metabolism in women. Br J Nutr 2003;90:993-995.

60 Arab L, Akbar J: Biomarkers and the measurement of fatty acids. Public Health Nutr 2002;5:865-871.
61 Ma J, Folsom AR, Shahar E, Eckfeldt JH: Plasma fatty acid composition as an indicator of habitual dietary fat intake in middleaged adults. Am J Clin Nutr 1995;62:564571.

62 Katan MB, van Birgelen A, Deslypere JP, Penders M, van Staveren WA: Biological markers of dietary intake, with emphasis on fatty acids. Ann Nutr Metab 1991;35:249252.

63 Glatz JF, Soffers AE, Katan MB: Fatty acid composition of serum cholesteryl esters and erythrocyte membranes as indicators of linoleic acid intake in man. Am J Clin Nutr 1989;49:269-276.
64 Plakké T, Berkel J, Beynen AC, Hermus RJ, Katan MB: Relationship between the fatty acid composition of the diet and that of the subcutaneous adipose tissue in individual human subjects. Hum Nutr Appl Nutr 1983; 37:365-372.

65 Beynen AC, Hermus RJ, Hautvast JG: A mathematical relationship between the fatty acid composition of the diet and that of the adipose tissue in man. Am J Clin Nutr 1980; 33:81-85. 